\title{
CT imaging of blunt chest trauma
}

\author{
Anastasia Oikonomou • Panos Prassopoulos
}

Received: 6 August 2010 /Revised: 28 November 2010 /Accepted: 27 January 2011 / Published online: 11 February 2011

(C) European Society of Radiology 2011

\begin{abstract}
Background Thoracic injury overall is the third most common cause of trauma following injury to the head and extremities. Thoracic trauma has a high morbidity and mortality, accounting for approximately $25 \%$ of traumarelated deaths, second only to head trauma. More than $70 \%$ of cases of blunt thoracic trauma are due to motor vehicle collisions, with the remainder caused by falls or blows from blunt objects.

Methods The mechanisms of injury, spectrum of abnormalities and radiological findings encountered in blunt thoracic trauma are categorised into injuries of the pleural space (pneumothorax, hemothorax), the lungs (pulmonary contusion, laceration and herniation), the airways (tracheobronchial lacerations, Macklin effect), the oesophagus, the heart, the aorta, the diaphragm and the chest wall (rib, scapular, sternal fractures and sternoclavicular dislocations). The possible coexistence of multiple types of injury in a single patient is stressed, and therefore systematic exclusion after thorough investigation of all types of injury is warranted.

Results The superiority of CT over chest radiography in diagnosing chest trauma is well documented. Moreover, with the advent of MDCT the imaging time for trauma patients has been significantly reduced to several seconds, allowing more time for appropriate post-diagnosis care.

Conclusion High-quality multiplanar and volumetric reformatted CT images greatly improve the detection of injuries
\end{abstract}

A. Oikonomou $(\square) \cdot$ P. Prassopoulos

Department of Radiology, University Hospital

of Alexandroupolis, Democritus University of Thrace,

Dragana, 68100 Alexandroupolis, Thrace, Greece

e-mail: aoikonom@med.duth.gr

P. Prassopoulos

e-mail: pprasopo@med.duth.gr and enhance the understanding of mechanisms of traumarelated abnormalities.

Keywords Blunt trauma $\cdot$ Lungs $\cdot \mathrm{CT}$

\section{Introduction}

Chest trauma is classified as blunt or penetrating, with blunt trauma being the cause of most thoracic injuries (90\%). The main difference lies in the presence of an opening to the inner thorax in penetrating trauma, created by stabbing or gunshot wounds, which is absent in blunt chest trauma [1]. Blunt thoracic injuries are the third most common injury in polytrauma patients following head and extremities injuries [2]. Although half of thoracic injuries are minor, 33\% require hospital admission [3]. Overall, blunt chest trauma is directly responsible for $25 \%$ of all trauma deaths [3] and is a major contributor in another $50 \%$ of trauma-related deaths. Moreover, chest trauma is the second most common cause of death, following only head trauma, and is by far the most common cause of death in the young age group between 15 and 44 years old [4]. Most blunt thoracic injuries are caused by motor vehicle crashes (MVC; 63$78 \%$ ), with the remainder (10-17\%) caused by falls from heights and a minority from blows from blunt objects or explosive devices [5].

Portable chest radiography is the initial imaging method used at the emergency workup of the polytrauma patient, and it is useful for detecting serious life-threatening conditions, such as a tension pneumothorax or haemothorax, mediastinal haematoma, flail chest or malpositioned tubes. However, the superiority of CT over chest radiography has been documented in the literature; CT detects significant disease in patients with normal initial radio- 
graphs and in $20 \%$ will reveal more extensive injuries compared with the abnormal initial radiographs, necessitating a change of management [6]. CT is far more effective than chest radiography in detecting pulmonary contusion, thoracic aortic injury and osseous trauma, especially at the cervicorthoracic spine. MDCT has dramatically decreased imaging times and offers readily available multiplanar reformatted images or more sophisticated volume-rendered and MIP images. Therefore, it has been established as the gold standard for the imaging evaluation of chest trauma and trauma in general [7].

This review focusses mainly on the typical CT findings as well as the pitfalls associated with the wide spectrum of types of injury in the thorax, including injury of the pleura (haemothorax, pneumothorax), the lung parenchyma (contusion, laceration, lung herniation and blast lung), the trachea and airways, the aorta, the heart and pericardium, the oesophagus, the diaphragm and the thoracic wall. The possible coexistence of multiple types of injury is stressed.

\section{Biomechanics of injury/trauma}

Four main mechanisms of injury are responsible for chest trauma: direct impact to the chest, thoracic compression, rapid acceleration/deceleration and blast injury.

Injuries from a direct impact are usually less dangerous and affect mainly the soft tissues of the chest wall (haematomas, rubbings). Occasionally, a localised injury to the osseous part of the chest wall can occur (rib fracture, sternal fracture and sternoclavicular dislocation) or, rarely, direct impact forces may be transmitted through the chest wall to the deeper organs, causing serious injury to the heart, lung or large mediastinal vessels.

In thoracic compression injuries intrathoracic structures strike a fixed anatomical structure - such as the chest or the spine-causing organ contusion or rupture. Thoracic compression may cause contusion or laceration of the lung parenchyma, pneumothorax or haemothorax, tracheobronchial fractures as well as rupture of the diaphragm.

In decelaration injuries the production of shearing forces causes direct compression against fixed points. This type is the most common and potentially lethal injury, and may cause major tracheobronchial disruption, cardiac contusions, aortic and diaphragmatic rupture [3].
Finally, with the increasing use of improvised explosive devices in terrorist attacks, blast injuries are occurring at an increasing rate. Explosion results from the instantaneous conversion of a solid or liquid material into gas after detonation of an explosive material. The blast pressure wave that is created exerts forces and pressure differentials mainly at air-tissue interfaces within the body, mostly affecting the pulmonary, gastrointestinal and auditory systems (primary blast injury). Secondary blast injuries result from objects propelled by the explosion, impacting the individual, while tertiary injuries follow when the individual is being propelled by the explosion $[8,9]$.

\section{CT protocols}

According to the type of CT available, a collimation of $1.25 \mathrm{~mm}$ (4-slice and 16-slice) or $0.6 \mathrm{~mm}$ (64-slice) is recommended. Use of $120 \mathrm{Kv}$ and $300 \mathrm{~mA}$ is acceptable [10], although attempts to reduce the radiation dose should be constantly pursued, especially if Automatic Exposure Control (AEC) can be applied wherever available [11]. Intravenous administration of contrast medium is imperative for imaging polytrauma patients, and as there is usually no luxury of time, only post-enhanced imaging is performed so as not to miss any injury of the major mediastinal vessels and the heart; optimal opacification may be obtained with injection of 100-140 ml of iodinated contrast medium at a flow rate of 3-4 ml/s and a delay of $25-40 \mathrm{~s}$. If $\mathrm{CT}$ of the thorax is part of a whole body trauma CT, then a compromise can be made with a 75-s delay for the whole body. When active bleeding is suspected, a delayed acquisition at $5 \mathrm{~min}$ is highly recommended, provided that the patient's haemodynamic stability allows for it (Table 1) $[12,13]$. Use of ECG gating for thoracic trauma is quite controversial, as although it may offer a higher diagnostic quality for any possible aortic, coronary or cardiac injury, it may on the other hand reduce the quality of bone and lung injury [14]. Given the fact that retrospective ECG gating compared with prospective ECG gating increases the radiation dose significantly, and that polytrauma patients may have an unstable heart rate higher than 80 beats $/ \mathrm{min}$, one should weigh the use of ECG gating carefully so as not to lose valuable time $[14,15]$. The axial thin slices can be used to create axial, coronal and sagittal reformations at 2

Table 1 CT protocols

\begin{tabular}{|c|c|c|c|c|c|c|c|}
\hline & Contrast medium & Collimation & $\mathrm{kVp}$ & mAs & Flow rate & Delay & Additional image \\
\hline Thoracic CT only & $120-140 \mathrm{ml}$ & $\begin{array}{l}4 \times 1.2516 \times 1.25 \\
64 \times 0.6\end{array}$ & $80-120$ & $300-400$ & $3-4 \mathrm{ml} / \mathrm{s}$ & $25-40 \mathrm{~s}$ & - \\
\hline $\begin{array}{l}\text { Thorax: part of whole } \\
\text { body CT }\end{array}$ & $120-140 \mathrm{ml}$ & $»$ & $»$ & $»$ & $»$ & $70-75 \mathrm{~s}$ & - \\
\hline Suspected extravasation & $120-140 \mathrm{ml}$ & $»$ & $»$ & $»$ & $»$ & $25-40$ or $75 \mathrm{~s}$ & $5 \mathrm{~min}$ \\
\hline
\end{tabular}


$2.5 \mathrm{~mm}$ for immediate viewing and exclusion of lifethreatening injuries. In the case of positive findings the images are transferred to a workstation for a more sophisticated imaging process and construction of maximum intensity projections (MIPs) or 3D aortogramarteriogram CT, 3D reformation of fractures or dislocations, and volume-rendered images for lung parenchyma or airway abnormalities [12, 13]. All reformatted images should be routinely viewed at soft-tissue, lung and bone windows.

\section{Pleura}

\section{Pneumothorax}

Trauma-related pneumothorax occurs in $30-40 \%$ of cases, and it is most commonly associated with rib fractures that lacerate the lung. Less commonly, pneumothorax may be caused by a disruption of closed airway spaces, such as the alveoli, due to a sudden increase in intrathoracic pressure or to a direct impact or deceleration force to the chest wall. Tracheobronchial injuries are also always associated with pneumothorax [5, 7, 16, 17]. CT is more sensitive in detecting pneumothoraces (Fig. 1), as $78 \%$ of them are nowadays believed to be missed on chest radiograph (occult pneumothoraces) $[18,19]$. Pneumothorax in supine polytrauma patients tends to accumulate at the anterior and

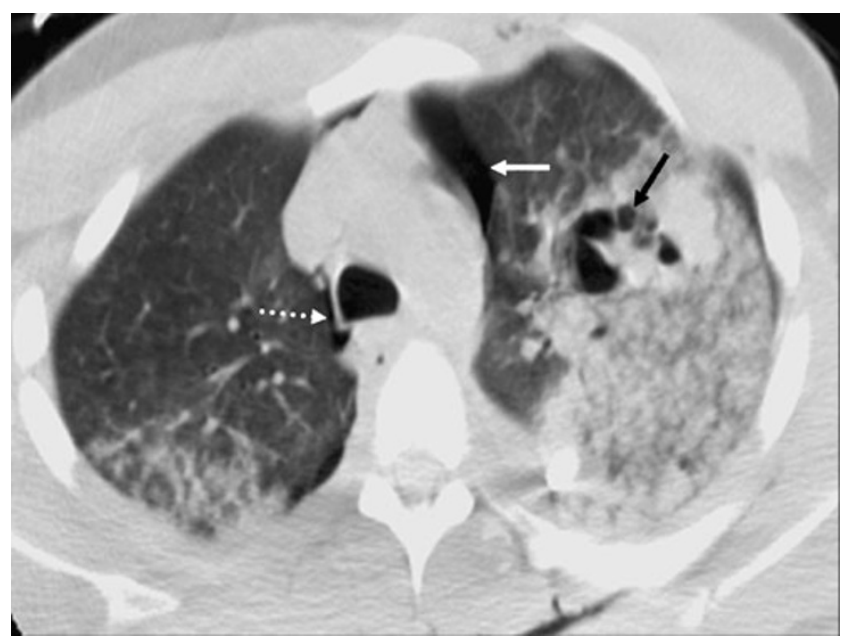

Fig. 1 Occult pneumothorax. Axial CT at lung window shows a small occult pneumothorax (missed on chest radiography) at the anteromedial part of the left upper lobe (white arrow). There are also bilateral ground-glass opacities and areas of consolidation in both upper lobes consistent with lung contusions. Note the presence of numerous small confluent pneumatoceles (Swiss cheese appearance) in the anterior segment of the left upper lobe, consistent with lung laceration (black arrow). Note also the presence of small pneumomediastinum (dotted white arrow) medial aspect of the lung, rendering it difficult to recognise on a supine chest radiograph, although it might be visible on an upright chest radiograph. Radiographic signs that may be present in the case of an occult pneumothorax include:

(1) Increased lucency at the affected hemidiaphragm,

(2) An abnormally deep costophrenic sulcus sign,

(3) A sharply defined radiolucent border of the mediastinum or heart, and

(4) The "double diaphragm sign" caused by the presence of air outlining the dome and insertion of the diaphragm $[7,20]$.

It is crucial to detect even a small pneumothorax in the trauma patient, as this can significantly enlarge under positive mechanical ventilation in the ICU or during general anaesthesia and endotracheal tube placement. Consequently, a prophylactic chest tube placement is considered [18] in small asymptomatic pneumothoraces $(<20 \%)$, although controversies exist about this practice in the literature [21]. However, there is growing evidence that occult pneumothorax can be safely treated without thoracostomy in non-ventilated patients [19].

Tension pneumothorax is an urgent clinical diagnosis where progressive accumulation of air-due to the onevalve mechanism - increases the intrathoracic pressure of the hemithorax involved, causing a contralateral shift of the mediastinum, compression of the superior vena cava and loss of venous return to the heart with resultant haemodynamic impairment. Chest radiography and CT will both show a contralateral shift in the mediastinum, hyperexpansion and hyperlucency of the ipsilateral lung with lung collapse towards the hilum, and inversion of the ipsilateral diaphragm (Fig. 2). Sudden evacuation of a large pneumothorax with tube drainage can be complicated by reexpansion pulmonary oedema, presenting the corresponding radiological signs. The complication is more common in younger patients (20-50 years of age) and occurs more often than was previously believed, and although it may be entirely asymptomatic, it has a reported variable mortality rate reaching $20 \%$ [22].

\section{Haemothorax}

Haemothorax occurs in $50 \%$ of chest trauma cases, with blood pooling into the pleural space from variable sources: the lung parenchyma, the chest wall, the great vessels, the heart or even the liver and spleen through diaphragmatic rupture [20]. Arterial bleeding (more commonly from the intercostal arteries, and the subclavian and internal mammary arteries) causes a more significant progressive increase in volume and mass effect compared with a 


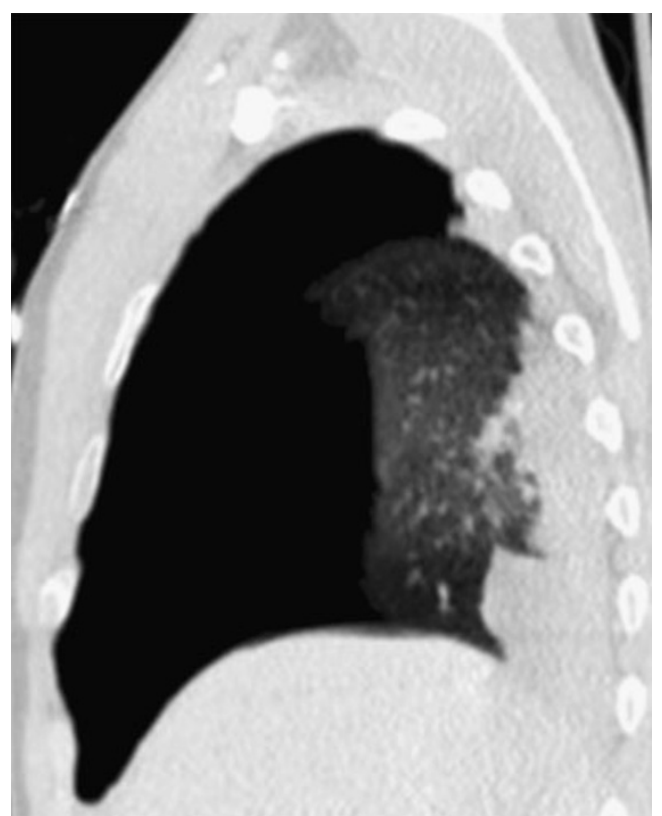

Fig. 2 Tension pneumothorax. Sagittal reformatted CT image at lung window showing tension pneumothorax with significantly collapsed lung at the posterior part of the hemithorax associated with ipsilateral pleural effusion

venous origin of haemorrhage [23]. Massive haemothorax occurs when the accumulation of blood in the pleural space exceeds 11 and is accompanied by haemodynamic impairment (Fig. 3) [2]. CT is very sensitive in detecting even a small haemothorax and can further characterise it by measuring accurately the Hounsfield (HU) units attenuation values of the pleural fluid. A reactive pleural effusion will have values not higher than $15 \mathrm{HU}$, while liquid blood will

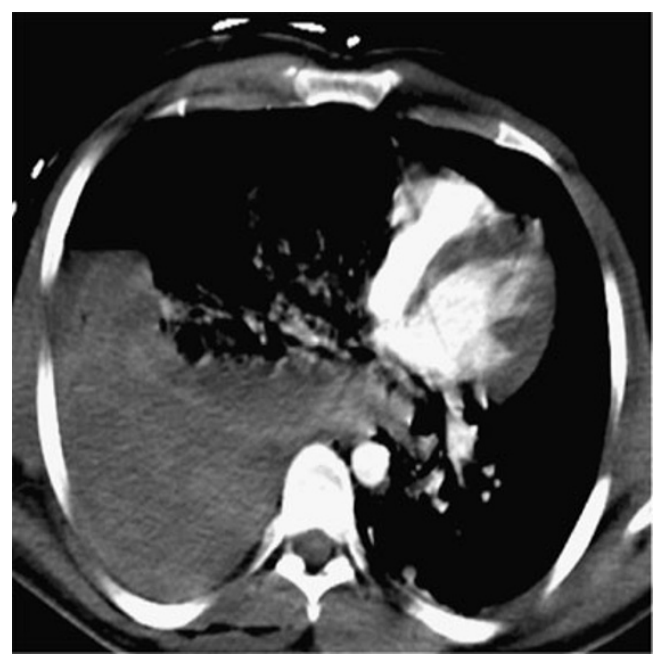

Fig. 3 Tension haemopneumothorax. Axial contrast-enhanced CT at mediastinal window shows a right tension haemopneumothorax with heterogeneous increased density due to presence of blood clots and a significant shift of the mediastinum contralaterally measure 30 to $45 \mathrm{HU}$, and the clotted blood should measure around 50-90 HU units [20, 24]. Occasionally a "haematocrit effect" is caused by the layering of different ages and statuses of coagulation of pleural blood products. In the case of active bleeding the fresh extravasated blood in contrast-enhanced CT may have attenuation values similar to the adjacent enhanced thoracic vessels $( \pm 10 \mathrm{HU})$ [17].

\section{Lung parenchyma}

\section{Pulmonary contusion}

Lung contusion is a focal parenchymal injury caused by disruption of the capillaries of the alveolar walls and septa, and leakage of blood into the alveolar spaces and interstitium [25]. It is the most common type of lung injury in blunt chest trauma with a reported prevalence of $17-70 \%$ [26]. The main mechanism is compression and tearing of the lung parenchyma at the site of impact (it may also occur contralaterally "contre-coup") against osseous structures, rib fractures or pre-existing pleural adhesions [27]. Lung contusion occurs at the time of injury, but it may be undetectable on chest radiography for the first $6 \mathrm{~h}$ after trauma. The pooling of haemorrhage and oedema will blossom at $24 \mathrm{~h}$, rendering the contusion radiographically more evident, although CT may readily reveal it from the initial imaging [28]. The appearance of consolidation on chest radiography after the first $24 \mathrm{~h}$ should raise suspicion of other pathological conditions such as aspiration, pneumonia and fat embolism [2]. Contusions appear as geographic, non-segmental areas of ground-glass or nodular opacities or consolidation on CT that do not respect the lobar boundaries and may manifest air bronchograms if the bronchioles are not filled with blood (Fig. 4) [17]. Subpleural sparing of 1-2 $\mathrm{mm}$ may be seen, especially in children (Fig. 4b) [29]. Clearance of an uncomplicated contusion begins at 24 to $48 \mathrm{~h}$ with complete resolution after 3 to 14 days [9]. Lack of resolution within the expected time frame should raise the suspicion of complications such as pneumonia, abscess or ARDS. Pulmonary contusion-despite the advances in prompt diagnosis with imaging and supportive management with critical care medicine - remains a predictor of ARDS and has a high mortality rate (10-25\%) [30].

\section{Pulmonary laceration}

Pulmonary laceration occurs in major chest trauma when disruption and tearing of the lung parenchyma follows shearing forces, caused by direct impact, compression or inertial deceleration [27]. Lung lacerations have been 
Fig. 4 Lung contusion. Axial $(\mathbf{a}, \mathbf{b})$ and coronal (c) CT images at lung window show nodular opacities of ground-glass opacity that do not respect the lung boundaries of the right upper lobe (white arrows) (a), diffuse areas of ground-glass opacity in the upper lobes bilaterally with subpleural sparing (white arrows) (b) and multiple areas of consolidation with air bronchograms (white arrows) and small lacerations (black arrows) in both lungs consistent with lung contusions. Note small bilateral pneumothorax in both lung apices (black dotted arrows) and cardiophrenic angles (black dotted arrows) (c)

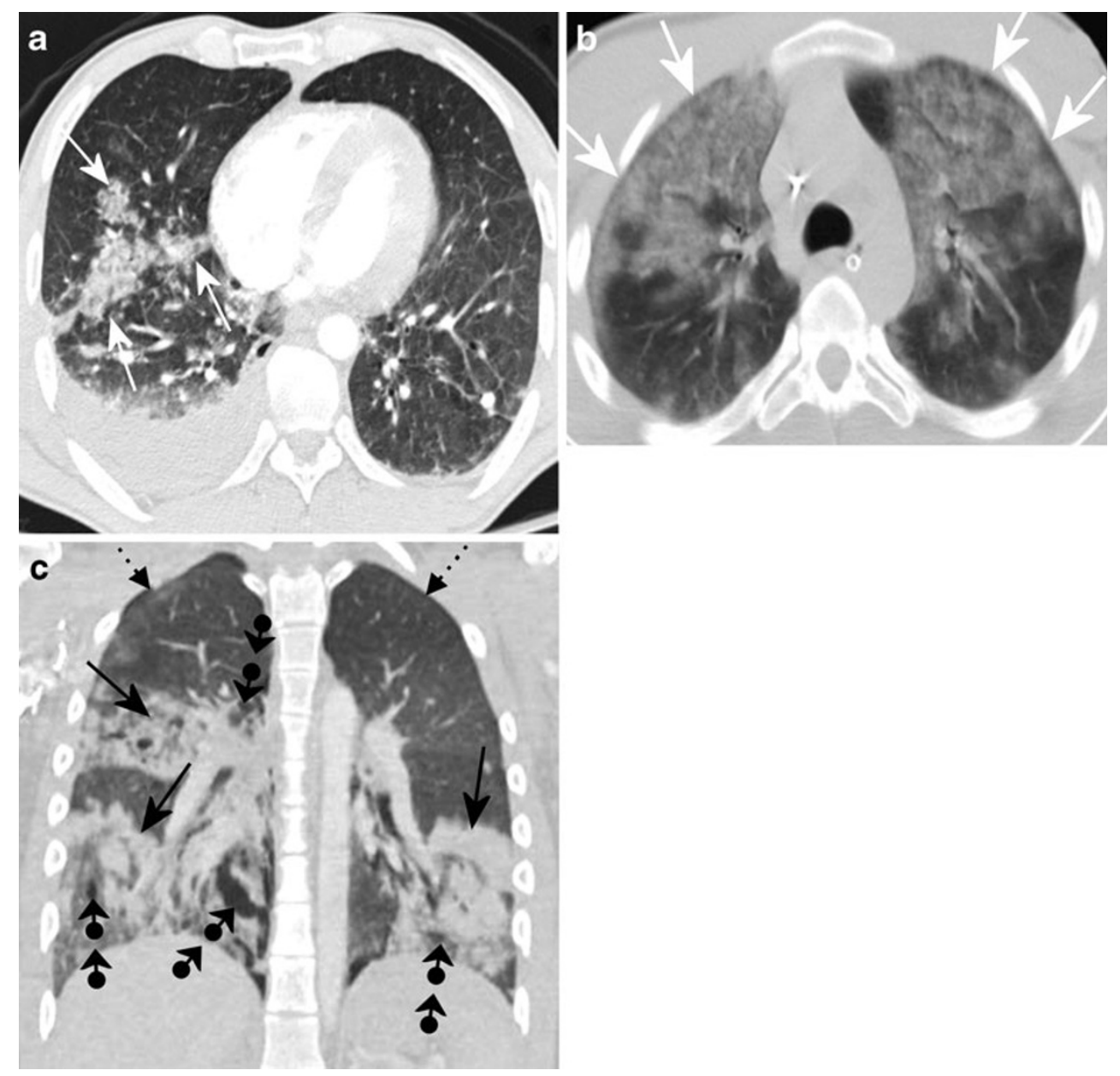

classified into the following four types according to the mechanism of injury [27]:

Type 1 Compression rupture injury (the most common type) is centrally located, can become very large and is produced by compression of the lung against the tracheobronchial tree.

Type 2 Compression shear injury is produced when the lower lobes are suddenly squeezed against the spine. It is located paraspinally and may be tubular in morphology (Fig. 5).

Type 3 Rib penetration tear is peripherally located, is small and round and is usually associated with pneumothorax (Fig. 6).

Type 4 The adhesion tear is seen adjacent to a previous pleuropulmonary adhesion and is almost always seen at surgery or at autopsy. Lung tissue surrounding a laceration retracts - because of the
Fig. 5 Lung laceration, type II. Coronal reformatted CT image at lung window (a) shows a lobulated paraspinal pneumatocele (arrow) surrounded by ground-glass opacity (contusion) in the right lung consistent with lung laceration (type II?). On mediastinal window lung laceration is seen to have been complicated by acute pulmonary embolism (dotted arrow)
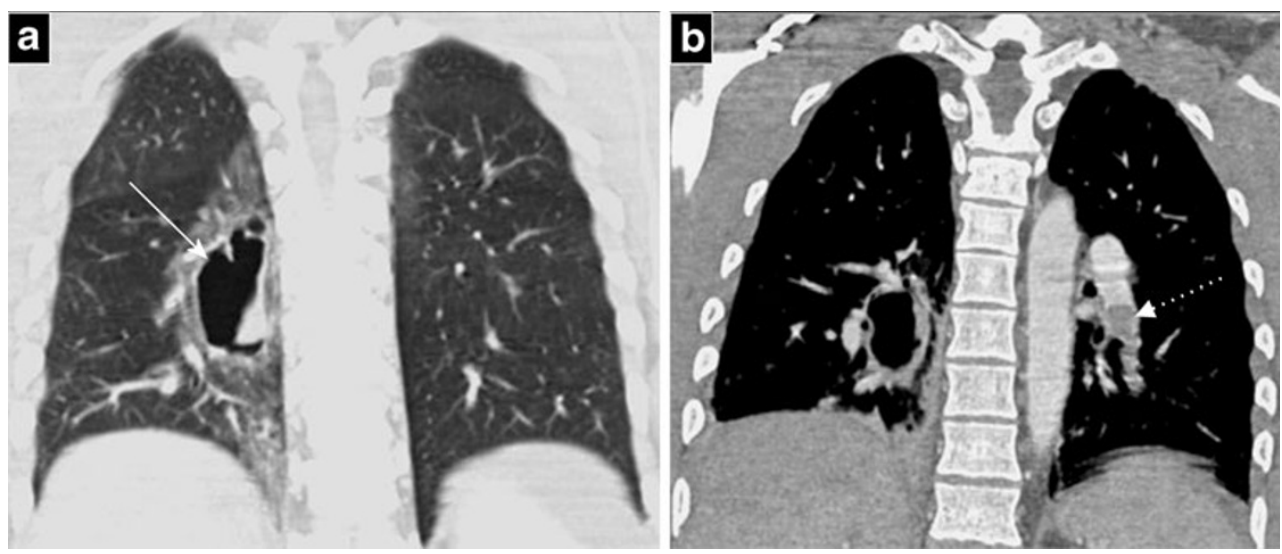


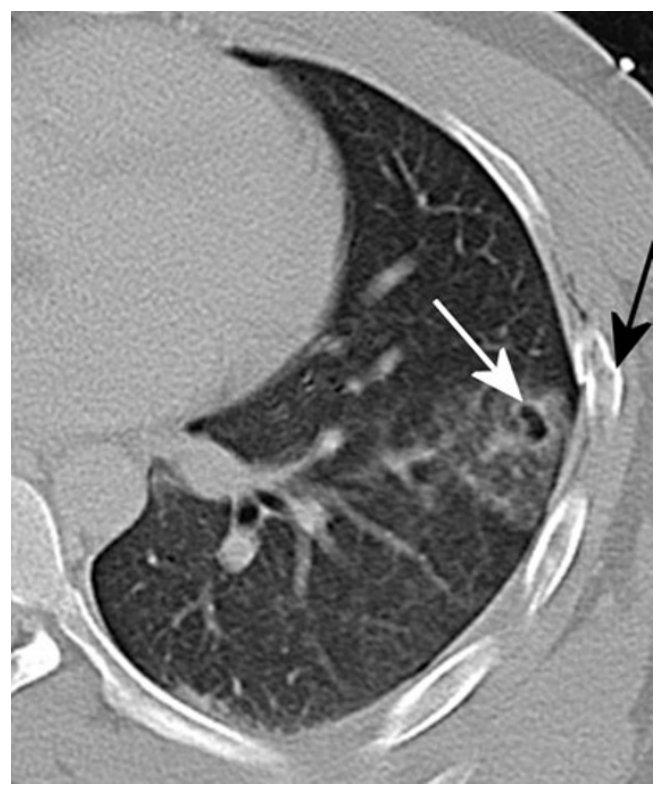

Fig. 6 Lung laceration, type IV. Axial CT image of the left lung at lung window shows a small peripheral laceration (white arrow) beneath a rib fracture (black arrow) surrounded by ground-glass opacity (lung contusion) and associated with a small ipsilateral pneumothorax

lung elastic recoil-leaving a round or oval cavity that may be filled with air (pneumatocele), blood (haematocele or haematoma) or both, creating an air-fluid level (haematopneumatocele). A laceration, although it may be filled with air, is usually surrounded by lung contusion and therefore is hidden on a chest radiograph during the first 23 days, until the contusion begins to resolve. CT, on the other hand, is significantly superior to chest radiography in detecting even a small laceration and in revealing the overall extent of the lacerations [27]. Lacerations (Fig. 1) may range from a solitary lesion to multiple confluent small ones presenting a "Swiss cheese appearance" [20]. Lacerations resolve more slowly than contusions, and clearance may take weeks or even months, and they may end in residual scarring [13]. Uncommonly, lacerations may be complicated by a pulmonary abscess, enlarge through a ball-valve mechanism or form a bronchopleural fistula [17], or it may be associated with acute pulmonary embolism (Fig. 5).

\section{Lung herniation}

Herniation of the lung parenchyma is an uncommon manifestation of blunt chest trauma, and it can occur through a congenital or a traumatic chest wall defect such

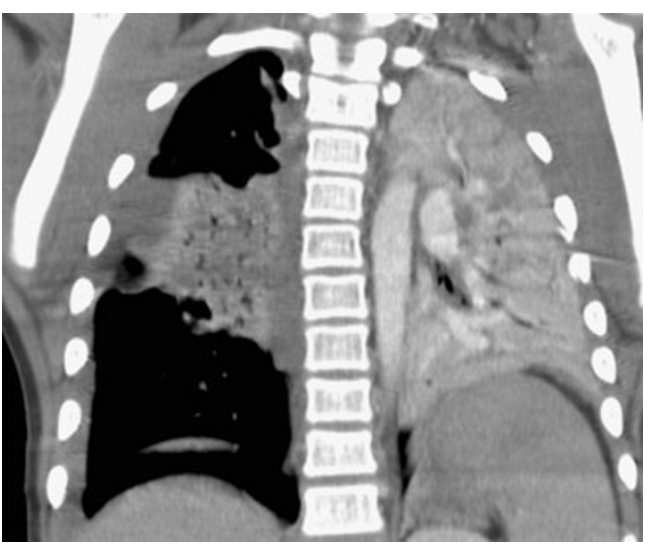

Fig. 7 Blast lung injury. Twenty-two-year-old patient who experienced the explosion of a grenade in his hands. Coronal CT reformatted contrast-enhanced CT image at mediastinal window shows bilateral perilar consolidations mimicking a butterfly or bat-wing appearance, consistent with blast lung. The left lung is almost completely collapsed, and there are bilateral haemothoraces

as multiple rib fractures or sternoclavicular or costochondral dislocations. Surgical repair is indicated when the patient is symptomatic or if the patient needs intubation and general anaesthesia as herniation may increase with positive-pressure ventilation $[24,31]$.

\section{Blast injury}

Blast lung is the most common fatal injury among initial survivors of explosions; $17-47 \%$ of people who die from explosions have had primary blast lung injury [8,9]. However, the in-hospital mortality rate for these patients ranges from 3.4 to $25 \%$ because of prompt diagnosis and aggressive treatment. The blast wave causes thoracic acceleration and propagates through lung parenchyma with subsequent severe disruption at the capillary-alveolar

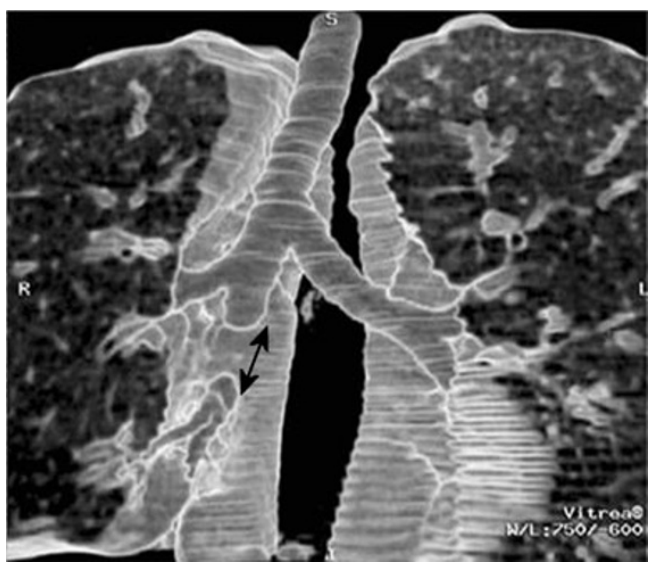

Fig. 8 Bronchial transection. A 22-year-old man involved in a car accident. Volume-rendered image of the tracheobronchial tree showing complete transection of the right intermediate bronchus (two-way arrow). (Courtesy of Dr Montserrat Bret, University Hospital La Paz, Madrid) 
Fig. 9 Pneumomediastinum. Axial CT images at wide lung window show pneumomediastinum with the presence of septae within the air in the anterior mediastinum (black arrows) (a), and in the middle and posterior mediastinum (black arrows) (b). Note also a right pneumothorax, bilateral lower lobe atelectases and subcutaneous emphysema (a) and a right haemopneumothorax and left pneumothorax (b)

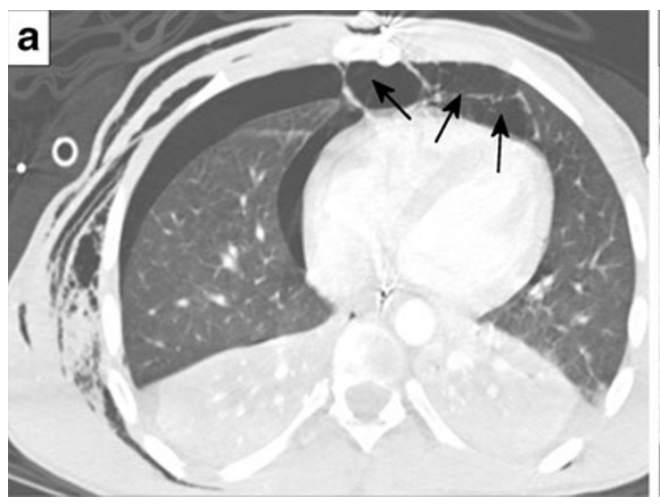

interface. This results in parenchymal haemorrhage and contusions, pulmonary oedema, pneumothorax, barotrauma and air embolism from arteriovenous fistulas, causing substantial immediate and delayed injury. Chest radiography and CT (Fig. 7) will reveal the "butterfly or batwing" pattern, representing central bilateral perihilar air space consolidation and ground-glass opacities that may contain air bronchograms [32].

\section{Airways}

\section{Trachea-bronchi}

Tracheobronchial injuries are rare, occurring in $0.2-8 \%$ of all cases of chest trauma. It is anticipated that the prevalence is higher, as $50 \%$ of patients die at the trauma scene within the first $2 \mathrm{~h}$ from associated injuries and

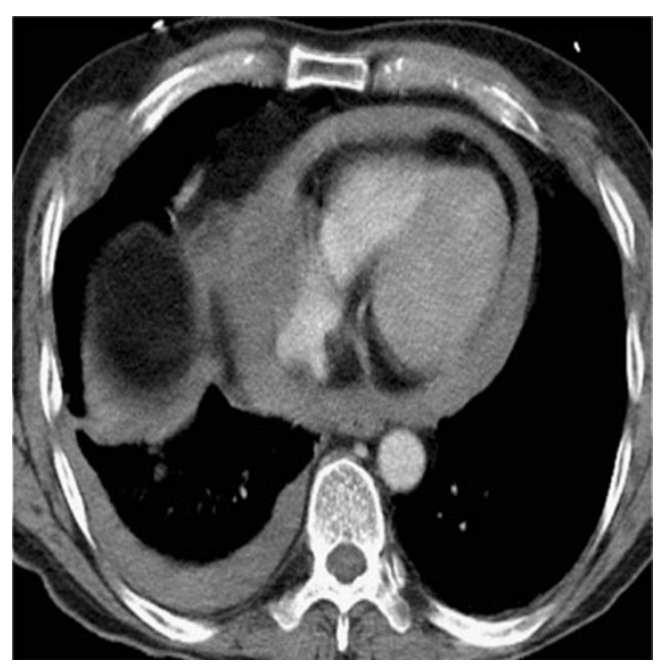

Fig. 10 Haemopericardium. Axial contrast-enhanced CT of the lower thorax at mediastinal window shows haemopericardium that may represent an indirect sign of pericardial or heart injury in a polytraumatised patient after a motor vehicle accident. There is also a small right haemothorax respiratory insufficiency $[2,33]$. They have a mortality rate of $30 \%$, and in two thirds of cases the diagnosis is delayed with subsequent serious complications, such as pneumonia, abscess, empyema, mediastinitis, sepsis, airway obstruction or atelectasis. Bronchial injuries occur more commonly than tracheal, usually on the right side and within $2.5 \mathrm{~cm}$ from the carina $[5,24]$, while $85 \%$ of tracheal lacerations occur $2 \mathrm{~cm}$ above the carina. Bronchial lacerations are usually parallel to the cartilage rings as opposed to tracheal ones that are vertical to the cartilage rings. A direct CT finding of tracheobronchial injuries is the cutoff of the tracheal and bronchial wall with extraluminal air surrounding the airway (Fig. 8). Indirect findings are the "fallen lung" sign, corresponding to the collapsed lung resting away from the hilum towards the dependent portion of the hemithorax [34], persistent pneumothorax after chest tube placement and herniation or overdistention of an endotracheal balloon if this is placed at the same level as the tracheal laceration [33]. Tracheal lacerations are usually associated with cervical subcutaneous emphysema. Tracheobronchial injuries in general are accompanied by pneumothorax and pneumomediastinum.

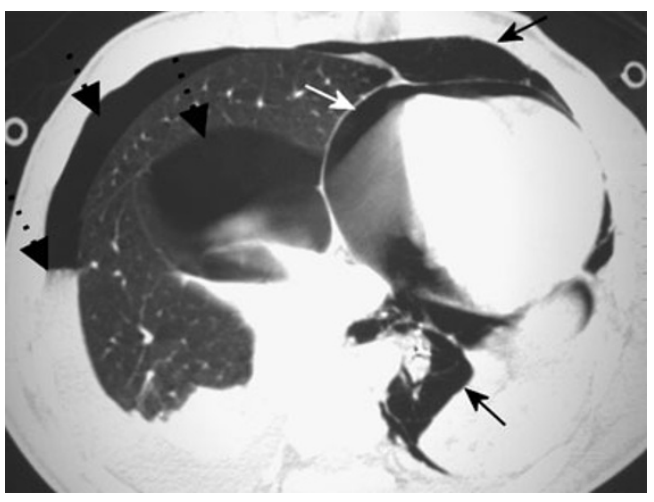

Fig. 11 Pneumopericardium. Axial CT image at lung window shows extensive pneumopericardium (white arrow), pneumomediastinum (black arrows), haemopneumothorax (black dotted arrows), collapsed left lung with ipsilateral shift of the mediastinum and collapse of the right lower lobe 

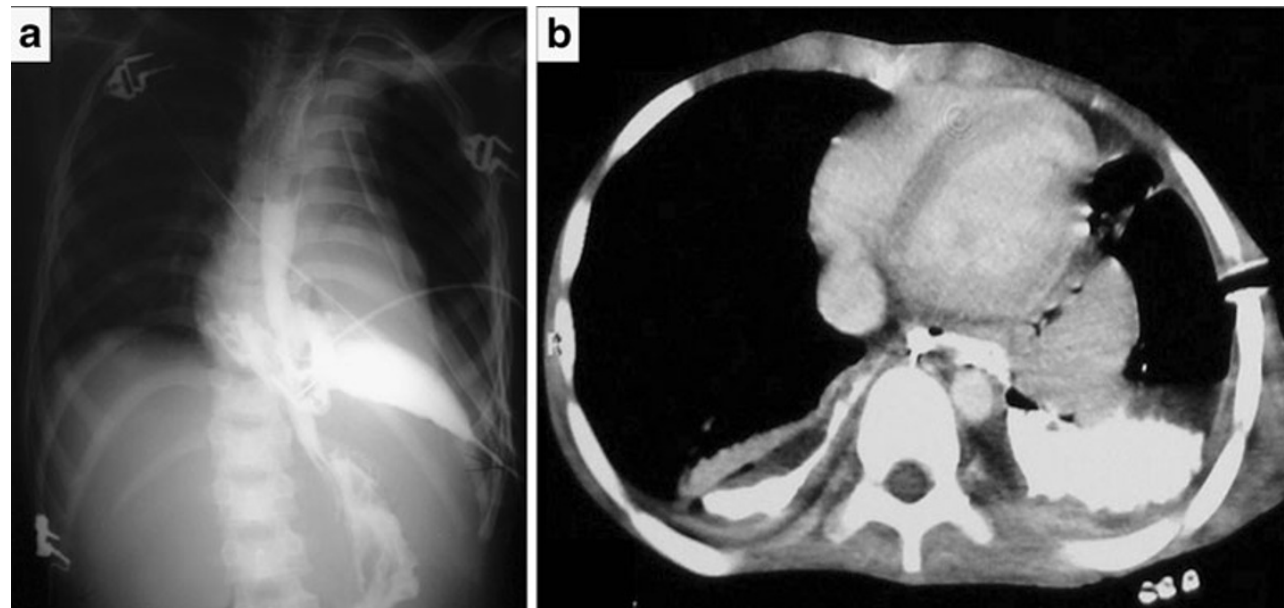

Fig. 12 Traumatic oesophageal rupture. A 12-year-old boy traumatised during a fall from a tree. Oesophagogram with per os administration of water-soluble contrast medium (a) shows leakage of contrast medium into both pleural spaces. Axial CT image of the thorax at the level of the lung bases (b) verifies the leakage of the

\section{Mediastinal structures}

Pneumomediastinum, the Macklin effect

Pneumomediastinum occurs in $10 \%$ of patients with blunt chest trauma, with less than $2 \%$ caused by blunt tracheobronchial injuries. Other sources of air originate from lung parenchymal injury, oesophageal injury, chest wall, neck and retroperitoneal injury. In a number of patients pneumomediastinum is attributed to the Macklin effect caused by alveolar ruptures that lead to air dissecting along broncho-

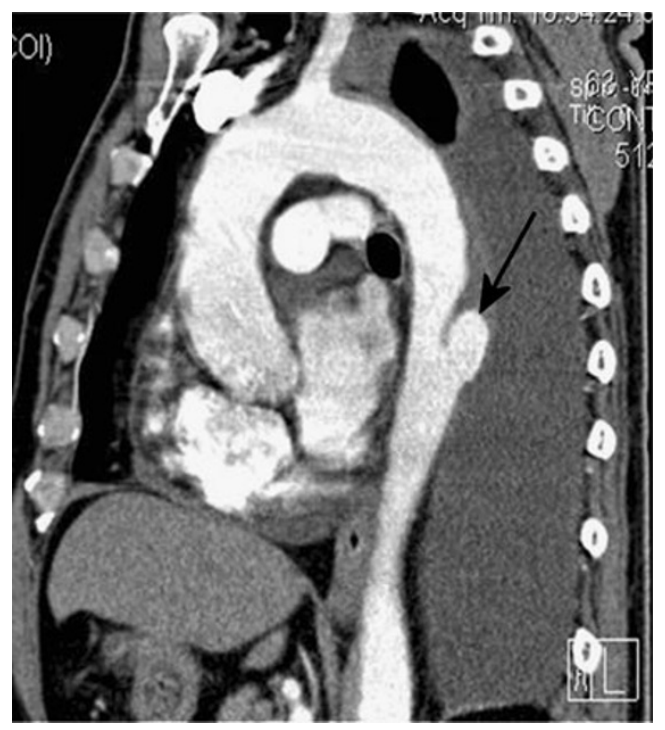

Fig. 13 Traumatic aortic pseudoaneurysm. Sagittal reformatted contrast-enhanced CT image of the thoracic aorta reveals a pseudoaneurysm of the greater curve of the mid-descending thoracic aorta (black arrow) contrast medium into the left and right pleural spaces. (Image reproduced from: Arora A, Puri SK, Upreti L, et al (2010). Oesophageal rupture: a rare complication of blunt trauma, \{Online . URL: http://www.eurorad.org/case.php?id=8447)

vascular bundles and spreading of the pulmonary interstitial emphysema into the mediastinum. Streaks of air surrounding and paralleling the bronchovascular bundles associated with pneumomediastinum may be observed on CT [35]. Pneumomediastinum may be mistaken for pneumothorax, but the presence of septae within it-delineated on wide lung window - may help in differentiating the two findings, especially if they coexist (Fig. 9).

Heart and pericardium

Cardiac injuries are the most lethal in chest trauma patients. They are more common in penetrating trauma, but they can occasionly occur in motor vehicle accidents and from

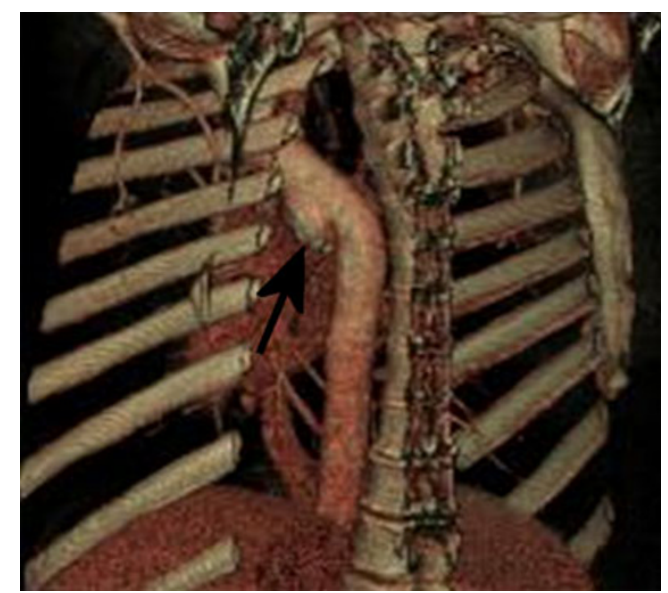

Fig. 14 Traumatic aortic pseudoaneurysm. Three-dimensional reconstructed CT image of the thoracic aorta shows a pseudoaneurysm of the inferior curve of the thoracic aorta immediately distal to the isthmus (arrow) 


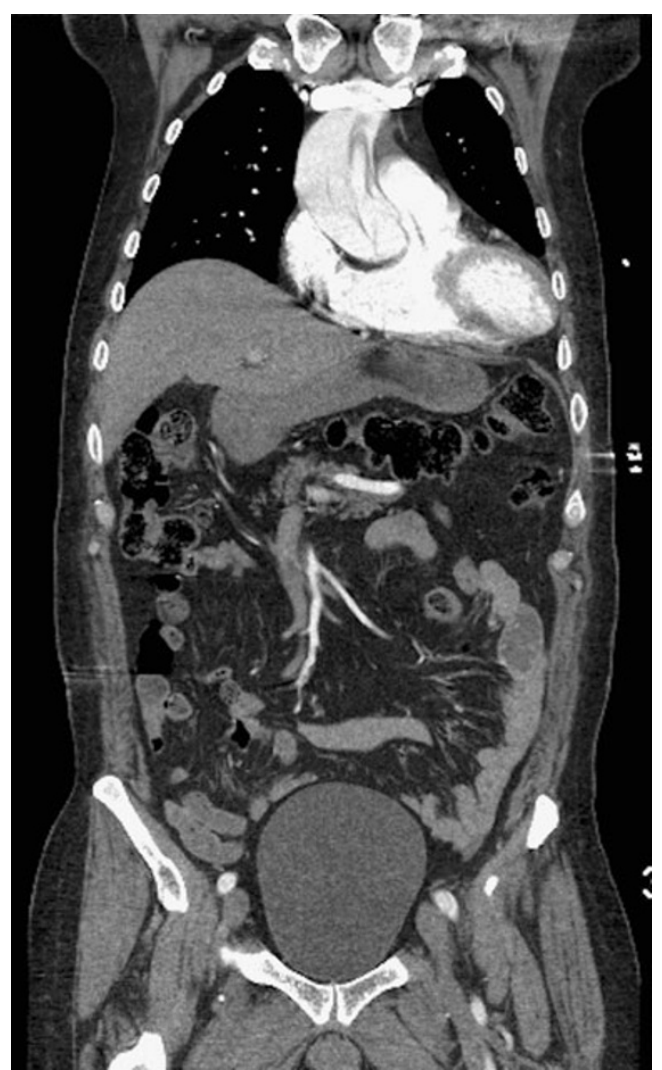

Fig. 15 Traumatic aortic dissection. Coronal contrast-enhanced CT reformatted image of the thorax and abdomen shows a traumatic dissection of the ascending thoracic aorta (Stanford type A)

severe blows to the anterior chest wall. Cardiac injury may be difficult to depict acutely in those who survive and should be treated with a high degree of clinical suspicion. It may range from a small focal contusion to a frank rupture of the heart, which is rare $(<2 \%)$, affecting mainly the right atrium [12]. CT has low sensitivity in detecting cardiac

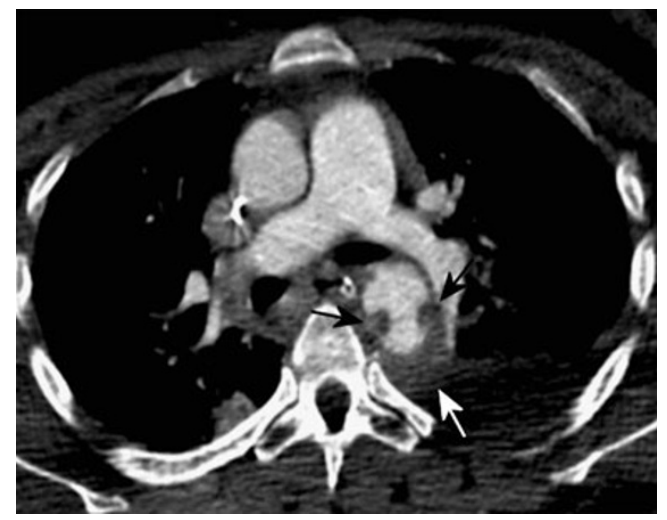

Fig. 16 Axial contrast-enhanced CT image shows thrombus protruding into the lumen of the descending thoracic aorta (black arrows), indicating aortic injury surrounded by periaortic haematoma (white arrow)

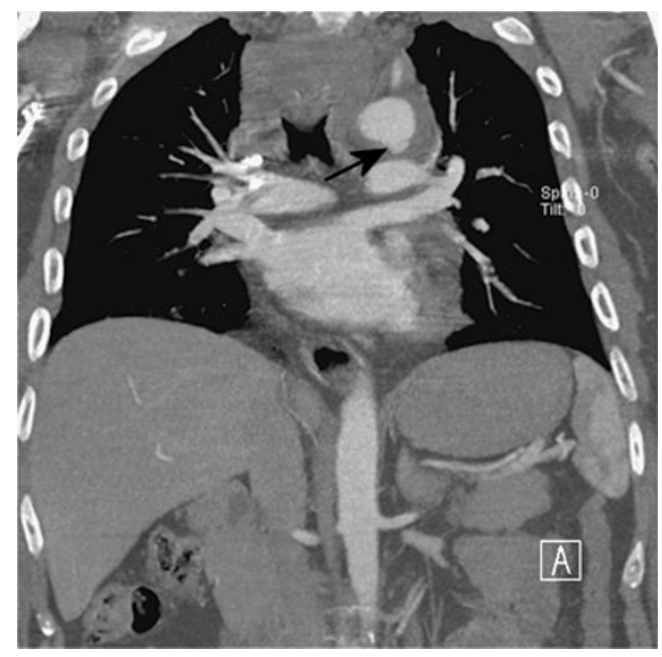

Fig. 17 Minimal aortic injury. Coronal MIP CT image of the thorax shows minimal aortic injury at the aortic isthmus (black arrow) involving only the intima, surrounded by periaortic haematoma

injury and may show haemo-pneumopericardium, cardiac contusion as a hypodense, non-enhancing focal area in the myocardium, contrast medium extravasation in the pericardial sac or the mediastinum, coronary artery laceration or valve injury [36]. Pericardial tears occur extremely rarely $(0.11 \%)$ and may range from tiny to the entire length of the pericardium. They usually manifest at the diaphragmatic surface of the pericardium on the left side. In cases of a large tear, intrapericardial cardiac herniation may occur, leading to death. Occasionally, there may also be intrapericardial bowel herniation [2]. On CT, the most common findings are haemopericardium (Fig. 10) and pneumopericardium (Fig. 11).

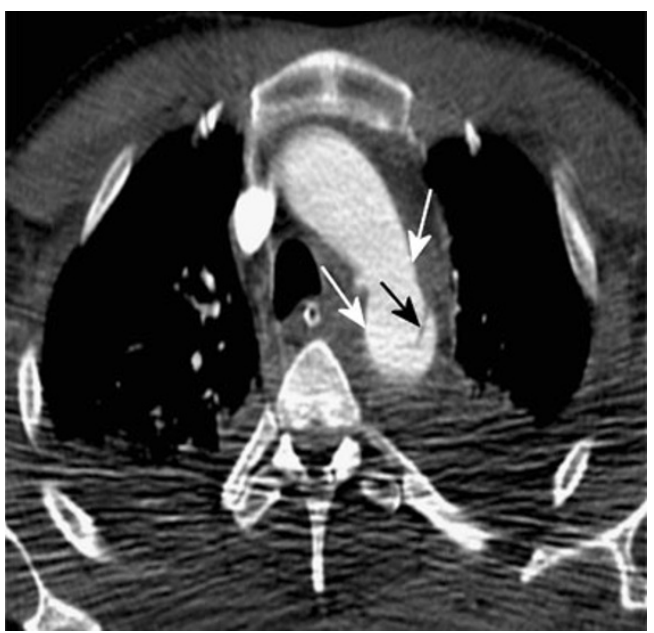

Fig. 18 Axial contrast-enhanced CT image shows presence of an intimal flap at the aortic arch (black arrow) surrounded by mediastinal haematoma with no preserved fat plane with the aorta (white arrows). Bilateral pleural haemothoraces are also seen 


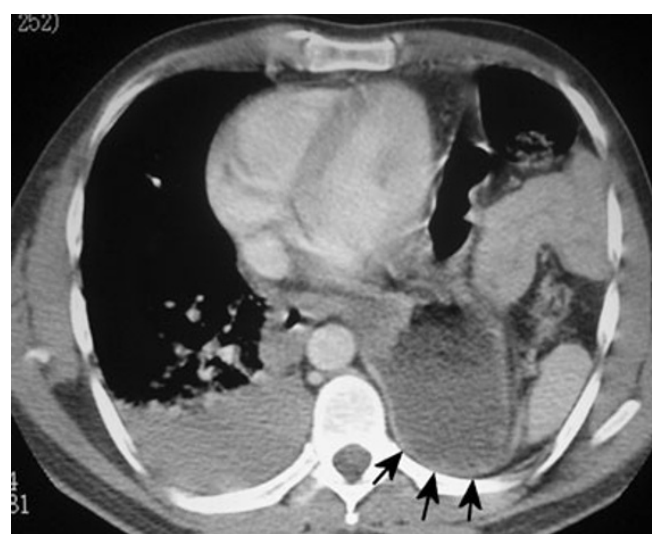

Fig. 19 Dependent viscera sign. Axial contrast-enhanced CT at the level of the lower lobes, at mediastinal window, shows intrathoracic presence of the stomach abutting the left posterior thoracic wall without intervening in the left hemidiaphragm (black arrows)

\section{Oesophagus}

Oesophageal injury is far more common in penetrating or iatrogenic injury and occurs only in $1 \%$ of cases of blunt chest trauma. The main mechanisms are a direct blow to the neck mainly affecting the cervical oesophagus, burst-type force or hyperextension injury affecting the distal oesophagus, or rupture by a vertebral body fracture [12]. On CT there will be mainly indirect findings of oesophageal rupture such as pneumomediastinum and peri-oesophageal air or abnormal mediastinal contour secondary to leakage of fluid, haematoma or mediastinitis. Hydropneumothorax is usually seen on the left side. Diagnosis can be confirmed (Fig. 12) by water-soluble contrast oesophagography showing leakage of contrast medium into the mediastinal or pleural space [37].

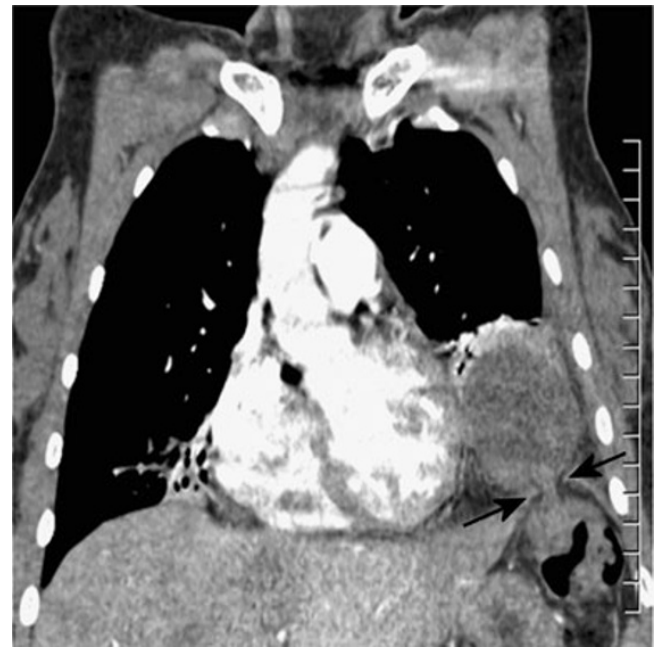

Fig. 20 The "hourglass" or "collar" sign of diaphragmatic rupture. Reformatted coronal contrast-enhanced CT image of the thorax shows waist-like stricture of the herniated left colon intrathoracically through the small defect of the left hemidiaphragm (black arrows)

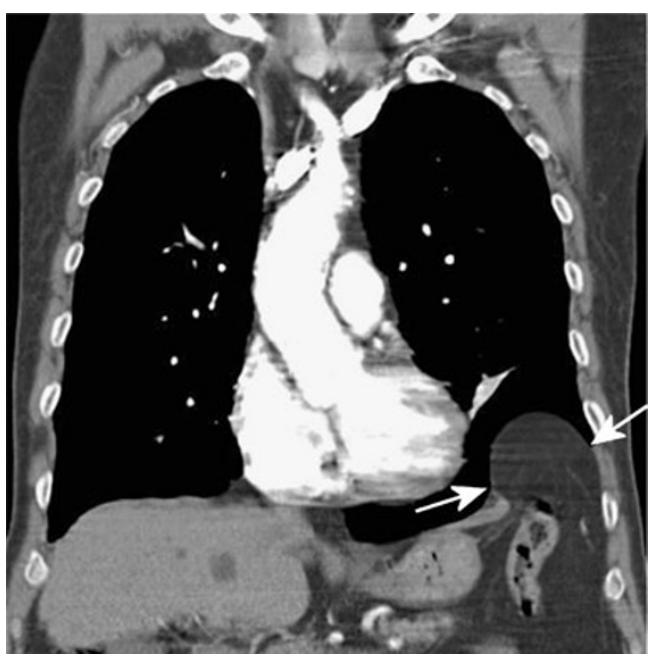

Fig. 21 Reformatted coronal contrast-enhanced CT image of the thorax shows intrathoracic herniation of intraperitoneal fat through the large defect of the left hemidiaphragm (white arrows)

Aorta, great vessels

Aortic injury occurs in $0.5 \%-2 \%$ of all non-lethal MVAs and is responsible for $10-20 \%$ of deaths in MVAs. Aortic injuries have a high morbidity and mortality; $90 \%$ of the patients die at the trauma scene, while $90 \%$ of initial survivors die within 4 months if the injury is undetected and untreated [38]. In the remaining $20 \%$ of cases, aortic injury is caused by falls and pedestrian injuries. Mechanisms of injury are variable and may overlap, including rapid deceleration, shearing forces, increased intravascular pressure caused by compression exceeding 2,000 $\mathrm{mmHg}$ (the water-hammer effect) or the "osseous pinch", which represents direct compression of the aorta between the anterior chest wall and the spine [38]. The most common site of injury is the isthmus, corresponding to $90-95 \%$ of

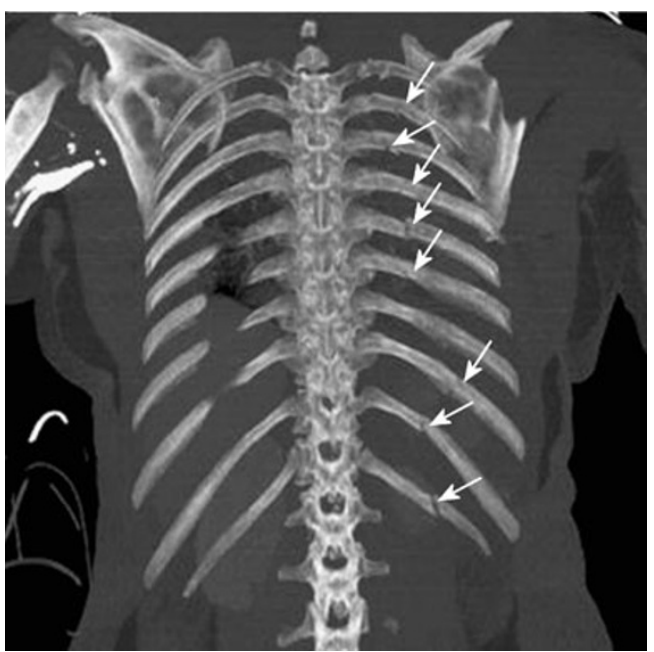

Fig. 22 Coronal MIP CT image showing multiple contiguous left rib fractures (arrows) 
Fig. 23 Coronal (a) and sagittal (b) reconstructed CT images show fractures of three contiguous right ribs (arrows) that were associated with paradox motion of the chest during respiration. Flail chest was suspected clinically and verified on imaging
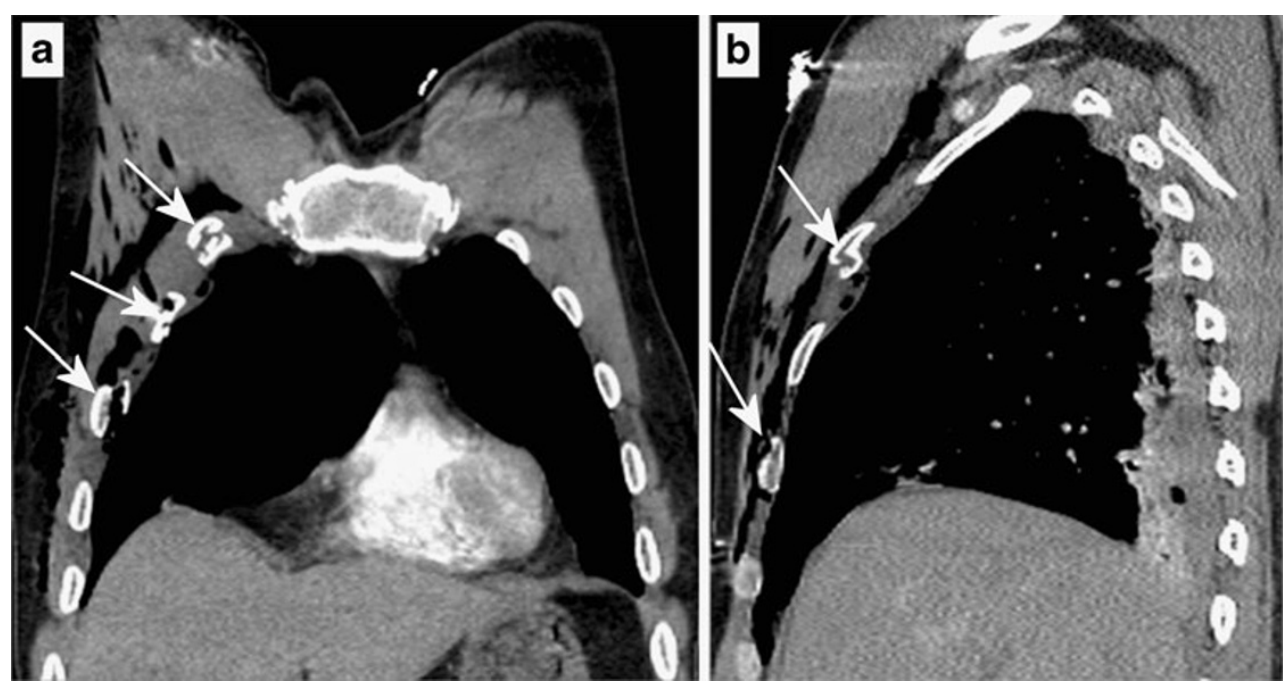

cases. Uncommon sites include the aortic root-ascending aorta, the aortic arch-branch vessels and the mid-distal descending aorta. The injury may be partial $(65 \%)$, involving only the intima and media, or transmural (35\%), also affecting the adventitia, which is lethal in almost all cases. The injury may be circumferential ( $45 \%$ of cases) or segmental (in $55 \%$ of cases), involving either the greater (Fig. 13) or the inferior curve (Fig. 14). CT may show direct and indirect signs. Direct signs include active contrast medium extravasation, dissection (Fig. 15), pseudoaneurysm (Figs. 13 and 14), intimal tear/flap (Fig. 16), thrombus protruding into the lumen (Fig. 17), and abrupt change in calibre (pseudocoarctation). Indirect CT signs are

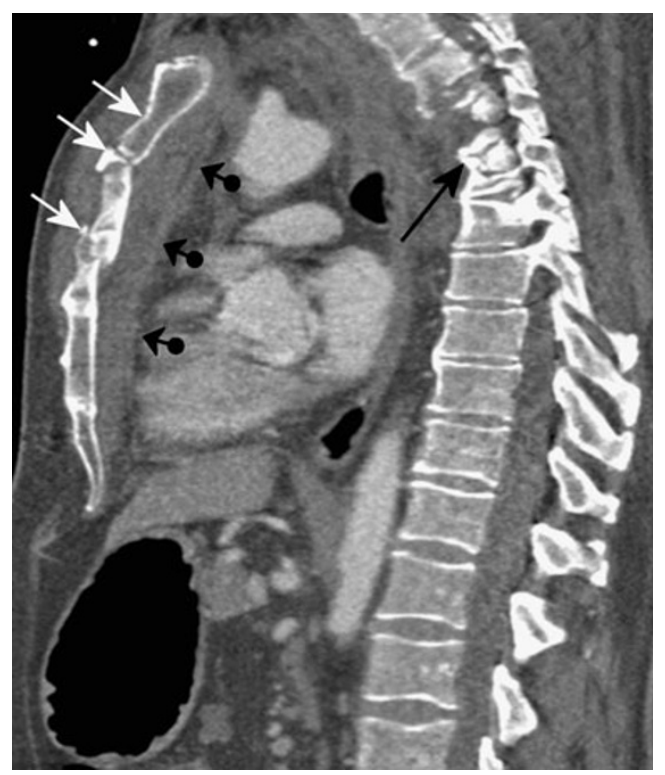

Fig. 24 Sternal fracture. Sagittal reconstructed CT image shows multiple fractures of the manubrium and the body of the sternum (white arrows) accompanied by extensive retrosternal haematoma (black ball arrows). Note also fracture of a thoracic vertebra (black arrow) indistinctness of mediastinal flat planes, periaortic haematoma and mediastinal haematoma (Figs. 16-18). Mediastinal haematoma is less than $20 \%$ predictive of aortic injury. In the absence of aortic injury, mediastinal haematoma may originate from venous injuries. In such cases the fat plane with the aorta is preserved, contrary to thoracic aortic injury, where haematoma develops in close contact with the aortic wall (Fig. 16-18). Minimal aortic injuries affect only the intima. They are diagnosed with increasing frequencybecause of improved MDCT technology - and constitute a diagnostic dilemma [39], as most of them remain stable or resolve on follow-up (Fig. 18). MDCT has very high sensitivity and specificity, reaching $98 \%$ and $100 \%$ accordingly for diagnosing aortic trauma. However, in studies where the presence of mediastinal haematoma (an indirect sign of aortic injury) was considered as a positive criterion for aortic injury, a significant number of false-positive

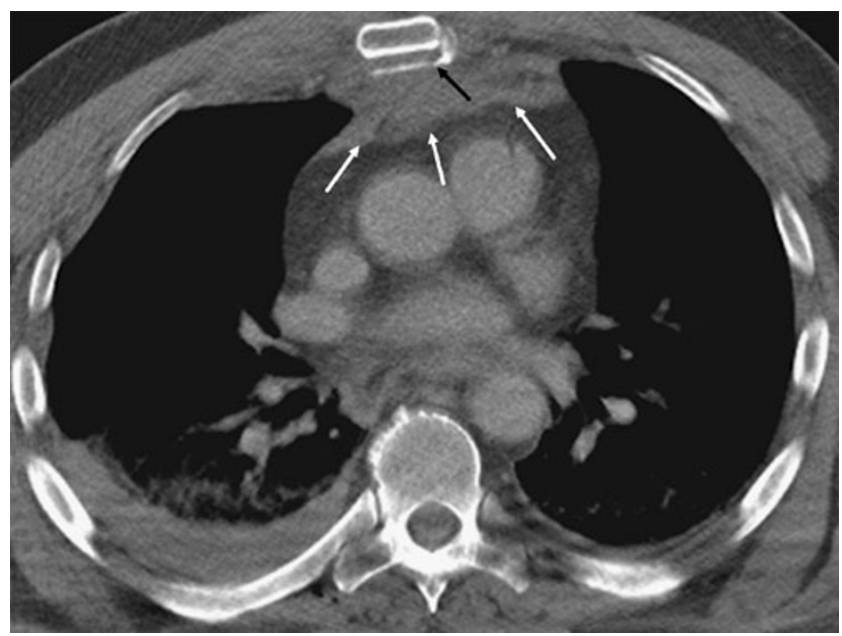

Fig. 25 Sternal fracture. Axial CT image at mediastinal window shows sternal fracture associated with retrosternal haematoma (black arrow). Note the preserved fat plane with the aorta, excluding the presence of aortic injury (white arrows) 


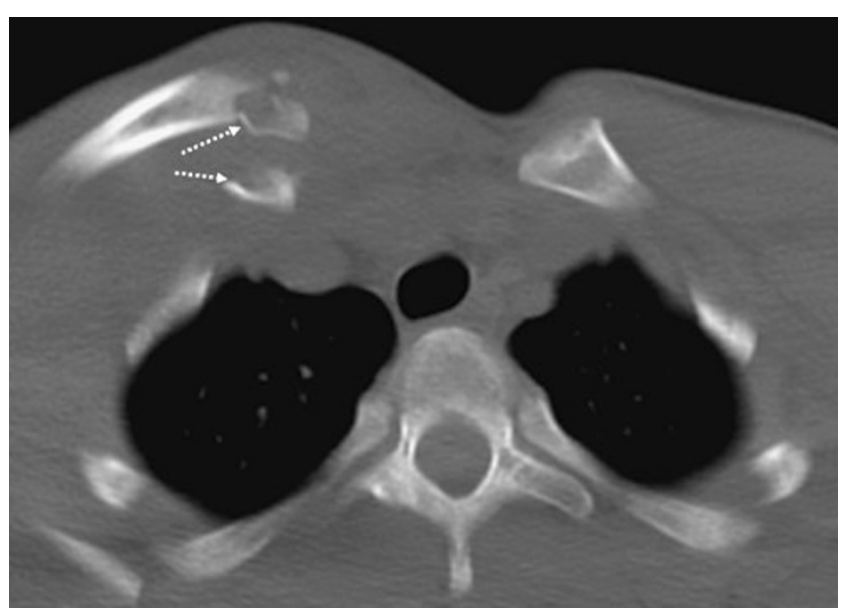

Fig. 26 Anterior sternoclavicular dislocation. Axial CT image shows clavicular fracture and anterior sternoclavicular dislocation (dotted arrows)

diagnoses occurred, and the specificity for aortic injury dropped significantly by up to $62 \%$. Nevertheless, MDCT has become the gold standard for ruling out aortic injury, and in those patients with unequivocal evidence of aortic injury, no further imaging is required [40]. No further workup is indicated if there is no direct evidence of aortic injury and no mediastinal haematoma on CT [38].

\section{Diaphragm}

Diaphragmatic injury occurs in $0.16 \%$ to $5 \%$ of blunt trauma cases, and it is more common in abdominal than in chest trauma. It is three times more common on the left side than on the right side, and the main mechanism is thought to be the sudden increase in intra-abdominal-thoracic

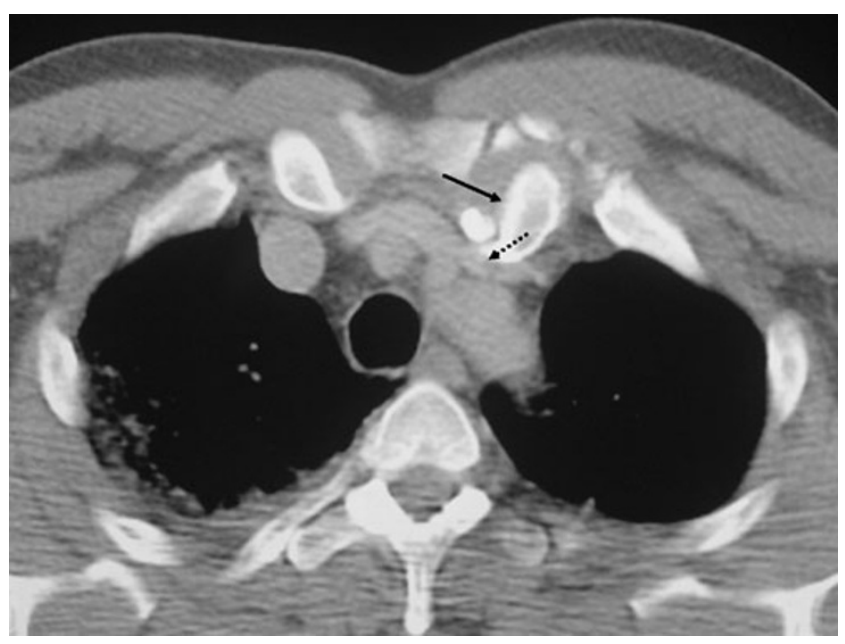

Fig. 27 Posterior sternoclavicular dislocation. Axial CT image shows posterior sternoclavicular dislocation (black arrow) associated with compression of the left innominate vein (black dotted arrow)

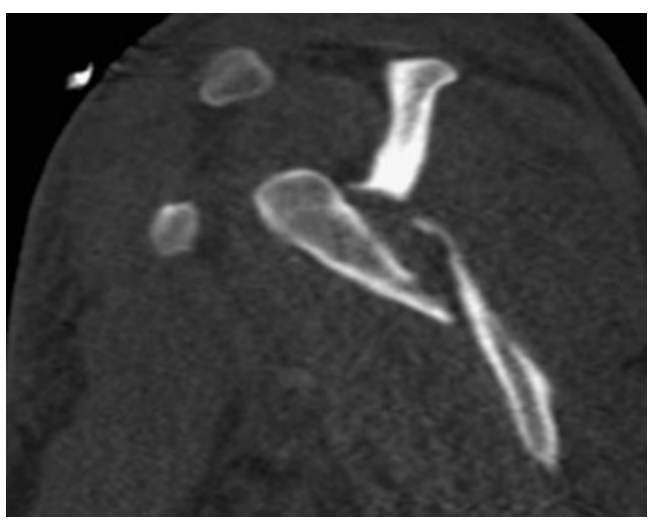

Fig. 28 Scapular fracture. Sagittal reconstructed CT image shows multiple fractures of the left scapula (arrows)
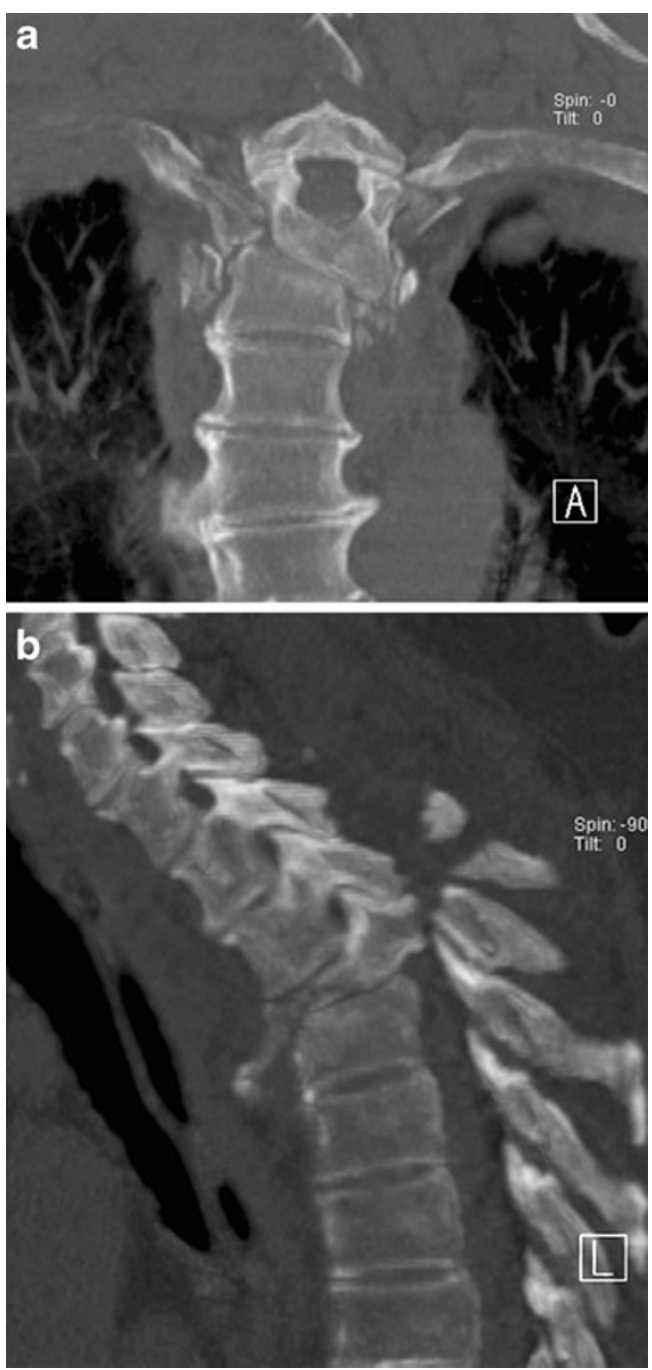

Fig. 29 Thoracic spine fracture. Coronal (a) and sagittal (b) CT reconstructed images of two different patients show fractures of the upper thoracic vertebrae with great detail 


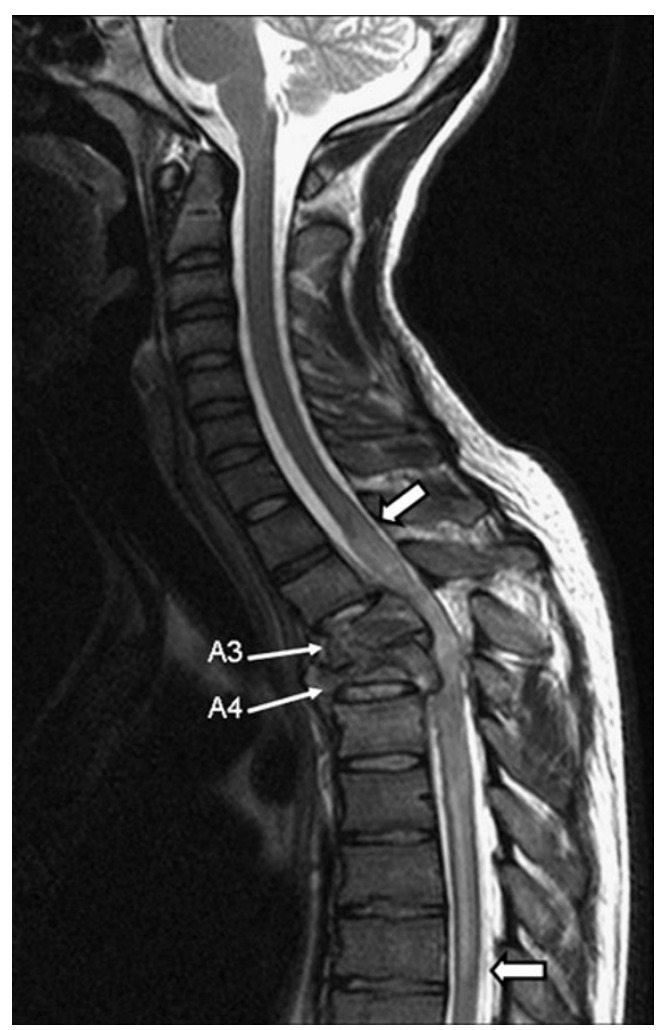

Fig. 30 Thoracic spine fracture and compressive myelopathy. Sagittal T2-weighted MRI of the cervicothoracic spine undertaken 1 week after a motor vehicle accident verifies the presence of extensive compressive myelopathy (between the two white arrows with black outline) due to fractures of the second and the third thoracic vertebrae (white arrows)

pressure against a fixed diaphragm. The most common site of rupture is at the posterolateral surface, at the site of embryonic diaphragmatic fusion. Through the diaphragmatic defect, depending on its size, there may be intrathoracic herniation of intra-abdominal visceral organs, which may be incarcerated, strangulated or perforated. CT findings of diaphragmatic rupture include [41] the diaphragmatic discontinuation and defect, the "collar sign" or "hourglass sign" formed by the waist-like stricture of partial intrathoracic herniation of the stomach or bowel (Fig. 19), and the "dependent viscera sign", which is formed by the posterior fall of the viscera towards and abutting the posterior dependent thoracic wall (with the patient supine) without the support of the intervening diaphragm (Fig. 20). Contrast-enhanced CT may reveal contrast material extravasation at the site of diaphragmatic rupture. Occasionally, there may be only peritoneal fat intrathoracically herniated through the defect (Fig. 21). Diaphragmatic injury is usually a delayed diagnosis because of other associated serious injuries that may mask the clinical symptoms. It has a high mortality rate (30\%) if it remains unrecognised [42]. The high-resolution coronal and sagittal reformations routinely produced with MDCT - compared with singlespiral CT-allow detection with high sensitivity, even of a small diaphragmatic defect.

\section{Thoracic wall}

\section{Soft tissue haematoma}

Soft tissue haematomas may occur during direct compression trauma when rib fractures cause laceration of veins or arteries. Soft tissue haematoma may become lifethreatening if the patient is under anticoagulant therapy. If it is arterial in origin, embolisation is indicated. Breast haematomas can be serious in direct impact or compression injuries [24].

\section{Ribs}

Rib fractures are the most common injury in blunt chest trauma, occurring in $50 \%$ of cases. A single rib fracture is usually not clinically significant, whereas multiple rib fractures indicate severe injury. Fractures of the first three ribs imply high-energy trauma that may be associated with injury of the brachial plexus or subclavian vessels. Fractures of the fourth up to the eighth ribs are the most common, while fractures of the last four ribs are usually associated with intra-abdominal injury. Reconstructed MIP

Table 2 Associated injuries

\begin{tabular}{ll}
\hline Sternal fracture & Heart injury \\
\hline Rib fracture & Pulmonary contusion, laceration \\
Upper rib fracture (first three ribs) & Brachial plexus, subclavian vessels \\
Lower rib fractures (last four ribs) & Intra-abdominal injury \\
Subcutaneous emphysema & Airway injury, oesophageal injury \\
Pneumomediastinum & Airway injury, lung injury, oesophageal injury \\
Sternoclavicular fracture (posterior sternoclavicular dislocation) & Mediastinal vessels, tracheal injury, oesophageal injury \\
Scapular fracture & Haemopneumothorax, lung injury, spine and clavicle fracture, subclavian \\
& vessels, brachial plexus \\
\hline
\end{tabular}


and volume-rendered $\mathrm{CT}$ images depict with great detail the number and sites of rib fractures (Fig. 22). Flail chest is a marker of significant intrathoracic injury with increased morbidity, in which three or more contiguous ribs are fractured in two or more sites (Fig. 23). The diagnosis is clinical based on the paradoxical motion during respiration, which may result in ventilatory compromise. More than $50 \%$ of cases require surgical treatment and prolonged mechanical ventilation [9].

\section{Sternum}

Sternal fractures have a prevalence of 3-8\% in blunt chest trauma. The main mechanism is deceleration injury or a direct blow to the anterior chest wall. It is considered a marker of cardiac contusion $(1.5 \%-6 \%)$. Sternal fractures are difficult to detect on lateral chest radiographs and even on axial CT images, as opposed to sagittal and coronal MDCT reformats, which have significant superiority (Fig. 24). It is almost always accompanied by anterior mediastinal haemorrhage, which has a preserved fat plane with the aorta (Fig. 25), as opposed to an anterior mediastinal haemorrhage secondary to aortic injury, which will present with a lost fat plane with the aorta [17].

Sternoclavicular dislocation is rare and occurs in $1-3 \%$ of all types of dislocation. Anterior sternoclavicular dislocation is more common and easily detectable, as it is palpable (Fig. 26). It usually has a benign course, but it implies a high-energy trauma and may be associated with haemopneumothorax, rib fractures or pulmonary contusion [43]. Posterior sternoclavicular dislocation is clinically and radiographically silent and carries serious morbidity, as it is associated with injuries of the mediastinal vessels, nerves, trachea and oesophagus (Fig. 27).

\section{Scapula}

Scapular fracture is uncommon, occurring in $3.7 \%$ of cases of blunt chest trauma. It is easily detected on initial radiographs and may be masked clinically by other associated serious injuries (Fig. 28). It indicates a highenergy force trauma with a direct blow to the scapula or force transmitted through the humerus. Associated injuries are pneumothorax, haemothorax, clavicular fracture and injuries of the lung parenchyma, subclavian vessels, brachial plexus or spine [44].

\section{Spine}

Thoracic spine fractures account for up to $30 \%$ of all spine fractures. Sixty-two percent of spine fractures will result in neurological deficits. The most vulnerable site is between the ninth and twelfth vertebra. The main mechanism is hyperflexion and axial loading. Plain radiographs may miss fractures of the spine and therefore may be unnecessary in those patients scheduled for CT [45]. Sagittal and coronal MDCT reformats (Fig. 29) readily reveal even small spinal fractures, whereas volume-rendered images are not helpful [12]. MDCT of the spine is highly indicated for spinal survey for possible fractures. However, in the case of suspected compressive myelopathy, MRI is the method of choice (Fig. 30).

Coexisting and associated injuries

It is important to remember that multiple types of injury in a single patient may coexist, and radiologists should not be disorientated by depicting one type of trauma and neglect other coexisting or associated types of injury (Table 2). Therefore, systematic exclusion after thorough investigation of all sites of possible injury in the thorax is warranted.

Acknowledgements The authors would like to thank the following radiologists for their valuable contributions: Aristi Kouri (Nicosia, Cyprus), Christoforos Schizas (Nicosia, Cyprus), Jean Seely (Ottawa, Canada), Rennae Thiessen (Vancouver, Canada), Argiro Voloudaki (Heraklion, Greece).

\section{References}

1. Shanmuganathan K, Matsumoto J (2006) Imaging of penetrating chest trauma. Radiol Clin North Am 44:225-238, Review

2. Kaewlai R, Avery LL, Asrani AV, Novelline RA (2008) Multidetector CT of blunt thoracic trauma. Radiographics 28:15551570

3. Scaglione M, Pinto A, Pedrosa I, Sparano A, Romano L (2008) Multi-detector row computed tomography and blunt chest trauma. Eur J Radiol 65:377-388

4. The American College of Surgeons Committee on Trauma Leadership (2007) In: Clark DE, Fantus RJ (eds) National Trauma Data Bank (NTDB) Annual Report 2007. American College of Surgeons, Chicago, IL, pp 1-64

5. Mayberry JC (2000) Imaging in thoracic trauma: the trauma surgeon's perspective. J Thorac Imaging 15:76-86

6. Exadaktylos AK, Sclabas G, Schmid SW, Schaller B, Zimmermann H (2001) Do we really need routine computed tomographic scanning in the primary evaluation of blunt chest trauma in patients with "normal" chest radiograph? J Trauma 51:1173-1176

7. Peters S, Nicolas V, Heyer CM (2010) Multidetector computed tomography-spectrum of blunt chest wall and lung injuries in polytraumatized patients. Clin Radiol 65:333-338, Review

8. Wolf SJ, Bebarta VS, Bonnett CJ, Pons PT, Cantrill SV (2009) Blast injuries. Lancet 374:405-415

9. Wanek S, Mayberry JC (2004) Blunt thoracic trauma: flail chest, pulmonary contusion, and blast injury. Crit Care Clin 20:71-81

10. Fanucci E, Fiaschetti V, Rotili A, Floris R, Simonetti G (2007) Whole body 16-row multislice CT in emergency room: effects of different protocols on scanning time, image quality and radiation exposure. Emerg Radiol 13:251-257

11. McCollough CH, Bruesewitz MR, Kofler JM Jr (2006) CT dose reduction and dose management tools: overview of available options. Radiographics 26:503-512, Review 
12. Rivas LA, Fishman JE, Múnera F, Bajayo DE (2003) Multislice CT in thoracic trauma. Radiol Clin North Am 41:599-616, Review

13. Novelline RA (2007) Imaging chest trauma. In: Diseases of the Heart, Chest \& Breast. Part 1. Springer, Milan

14. Schertler T, Glücker T, Wildermuth S, Jungius KP, Marincek B, Boehm T (2005) Comparison of retrospectively ECG-gated and nongated MDCT of the chest in an emergency setting regarding workflow, image quality, and diagnostic certainty. Emerg Radiol 12:19-29

15. Bruzzi JF, Rémy-Jardin M, Delhaye D, Teisseire A, Khalil C, Rémy J (2006) When, why, and how to examine the heart during thoracic CT: Part 1, basic principles. AJR Am J Roentgenol 186:324-332, Review

16. Lomoschitz FM, Eisenhuber E, Linnau KF, Peloschek P, Schoder M, Bankier AA (2003) Imaging of chest trauma: radiological patterns of injury and diagnostic algorithms. Eur J Radiol 48:61-70

17. Miller LA (2006) Chest wall, lung, and pleural space trauma. Radiol Clin North Am 44:213-224

18. McGillicuddy D (2007) Diagnostic dilemmas and current controversies in blunt chest trauma. Emerg Med Clin North Am 25:695711

19. Ball CG, Kirkpatrick AW, Laupland KB, Fox DI, Nicolaou S, Anderson IB, Hameed SM, Kortbeek JB, Mulloy RR, Litvinchuk S, Boulanger BR (2005) Incidence, risk factors, and outcomes for occult pneumothoraces in victims of major trauma. J Trauma 59:917-924

20. Mirvis SE (2005) Imaging of acute thoracic injury: the advent of MDCT screening. Semin Ultrasound CT MR 26:305-331

21. Brasel KJ, Stafford RE, Weigelt JA, Tenquist JE, Borgstrom DC (1999) Treatment of occult pneumothoraces from blunt trauma. J Trauma 46:987-990

22. Kim YK, Kim H, Lee CC, Choi HJ, Lee KH, Hwang SO, Oh JH, Lee YH, Singer AJ (2009) New classification and clinical characteristics of reexpansion pulmonary edema after treatment of spontaneous pneumothorax. Am J Emerg Med 27:961-967

23. Shanmugathan K, Mirvis SE (1999) Imaging diagnosis of nonaortic thoracic injury. Radiol Clin North Am 37:533-551

24. Sangster GP, González-Beicos A, Carbo AI, Heldmann MG, Ibrahim H, Carrascosa P, Nazar M, D'Agostino HB (2007) Blunt traumatic injuries of the lung parenchyma, pleura, thoracic wall, and intrathoracic airways: multidetector computer tomography imaging findings. Emerg Radiol 14:297-310

25. Wicky S, Wintermark M, Schnyder P, Capasso P, Denys A (2000) Imaging of blunt chest trauma. Eur Radiol 10:1524-1538, Review

26. Gavelli G, Canini R, Bertaccini P, Battista G, Bna C, Fattori R (2002) Traumatic injuries: imaging of thoracic injuries. Eur Radiol 12:1273-1294

27. Wagner RB, Crawford WO Jr, Schimpf PP (1988) Classification of parenchymal injuries to the lung. Radiology 167:77-82
28. Schild HH, Strunk H, Weber W, Stoerkel S, Doll G, Hein K, Weitz M (1989) Pulmonary contusion: CT vs plain radiograms. J Comput Assist Tomogr 13:417-420

29. Donnelly LF, Klosterman LA (1997) Subpleural sparing: a CT finding of lung contusion in children. Radiology 204:385-387

30. Miller PR, Croce MA, Bee TK, Qaisi WG, Smith CP, Collins GL, Fabian TC (2001) ARDS after pulmonary contusion: accurate measurement of contusion volume identifies high-risk patients. J Trauma 51:223-230

31. Clark AJ, Hughes N, Chisti F (2009) Traumatic extrathoracic lung herniation. Br J Radiol 82:e82-e84

32. Avidan V, Hersch M, Armon Y, Spira R, Aharoni D, Reissman P, Schecter WP (2005) Blast lung injury: Clinical manifestations, treatment and outcome. Am J Surg 190:927-931

33. Scaglione M, Romano S, Pinto A, Sparano A, Scialpi M, Rotondo A (2006) Acute tracheobronchial injuries: Impact of imaging on diagnosis and management implications. Eur J Radiol 59:336-343

34. Tack D, Defrance P, Delcour C, Gevenois PA (2000) The CT fallen-lung sign. Eur Radiol 10:719-721

35. Wintermark M, Schnyder P (2001) The Macklin effect: a frequent etiology for pneumomediastinum in severe blunt chest trauma. Chest 120:543-547

36. Sliker CW, Mirvis SE, Shanmuganathan K, Meyer CA (2000) Blunt cardiac rupture: value of contrast-enhanced spiral CT. Clin Radiol 55:805-808

37. de Lutio di Castelguidone E, Merola S, Pinto A, Raissaki M, Gagliardi N, Romano L (2006) Esophageal injuries: spectrum of multidetector row CT findings. Eur J Radiol 59:344-348

38. Steenburg SD, Ravenel JG, Ikonomidis JS, Schönholz C, Reeves S (2008) Acute traumatic aortic injury: imaging evaluation and management. Radiology 248:748-762, Review

39. Malhotra AK, Fabian TC, Croce MA, Weiman DS, Gavant ML, Pate JW (2001) Minimal aortic injury: a lesion associated with advancing diagnostic techniques. J Trauma 51:1042-1048

40. Mirvis SE (2006) Thoracic vascular injury. Radiol Clin North Am 44:181-197

41. Bergin D, Ennis R, Keogh C, Fenlon HM, Murray JG (2001) The "dependent viscera" sign in CT diagnosis of blunt traumatic diaphragmatic rupture. AJR Am J Roentgenol 177:1137-1140

42. Mirvis SE, Shanmuganagthan K (2007) Imaging hemidiaphragmatic injury. Eur Radiol 17:1411-1421

43. Cope R (1993) Dislocations of the sternoclavicular joint. Skeletal Radiol 22:233-238

44. Weening B, Walton C, Cole PA, Alanezi K, Hanson BP, Bhandari M (2005) Lower mortality in patients with scapular fractures. J Trauma 59:1477-1481

45. Rhea JT, Sheridan RL, Mullins ME, Nonelline RA (2001) Can chest and abdominal trauma CT eliminate the need for plain films of the spine? - Experience with 329 multiple trauma patients. Emerg Radiol 8:99-104 